Finisterra, XXXIII, 65, 1998, pp. 39-50

\title{
LA MOBILITÉ DU REGARD ET SON INSTRUMENTALISATION. FRANZ SCHRADER À LA CROISÉE DE L'ART ET DE LA SCIENCE
}

VINCENT BERDOULAY ${ }^{1}$

HELENE SAULE-SORBE ${ }^{2}$

Résumé: L'étude de la contribution de Franz Schrader souligne l'intérêt de revisiter l'histoire de la pensée géographique en fonction du thème de la mobilité du regard et de son instrumentalisation, particulièrement à la lumière des interactions entre l'art et la science.

Mots-clés: Regard géographique, paysage, sujet, outil technique, art, Franz Schrader.

\begin{abstract}
THE MOBILITY AND THE INSTRUMENTATION OF THE Eye LOOKING AT THE LANDSCAPE. FRANK SCHRADER IN THE INTERSECTION BETWEEN ART AND SCIENCE - The study of the work of Franz Schrader underlines the interest of revisiting the history of geographical thought according to the theme of the mobility of the eye looking at the landscape. This is particularly interesting in the light of the interactions between art and science. The issue of how this visual perspective is made instrumental is emphasised, with Schrader's orograph as a key-example.
\end{abstract}

Key-words: The geographical ways of seeing, landscape, self, technical instrument, art, Franz Schrader.

Resumo: A MOBILIDADE DO OLHAR E A SUA INSTRUMENTALIZAÇÃO. FrANZ SCHRADER NO CRUZAMENTO DA ARTE E DA CIÊNCIA - O estudo da contribuição de Franz Schrader sublinha o interesse de revisitar a história do pensamento geográfico, em função do tema da mobilidade do olhar e da sua instrumentalização, particularmente à luz das interacções entre a arte e a ciência.

Palavras-chave: Olhar geográfico, paisagem, sujeito, instrumento técnico, arte, Franz Schrader.

\footnotetext{
1 Professeur de géographie, Université de Pau et des Pays de l'Adour.

2 Maître de conférences d'arts plastiques, Université de Bordeaux III.

Chercheurs au Laboratoire SET (CNRS), Domaine universitaire, 64000 Pau (France).

Tel.: (33) 5599231 23; Fax: (33) 5598083 39; courrier: set@univ-pau.fr
} 
La connaissance géographique dépend de l'expérience du déplacement, nécessaire à la satisfaction de la curiosité pour les lieux. Si le voyage d'exploration en semble l'archétype, c'est l'idée plus générale de la mobilité du regard qui la fonde. Celui-ci s'exerce en effet lors de déplacements qui engagent le corps mais qui sont aussi le fruit de l'activité cognitive. A la mobilité dans l'espace géographique concret fait écho celle qui se déploie dans l'espace des représentations, d'un domaine du savoir à un autre. Or la mobilité du regard repose sur l'instrumentalisation qui lui est donnée. Ainsi, au delà de la logistique du voyage et des procédés de notation de ce qui est vu, il faut se pencher sur la démarche même qui fonde l'intérêt pour la mobilité du regard et qui l'instrumentalise en conséquence. C'est l'objectif des pages qui suivent. Ce faisant, on verra que l'histoire de la pensée géographique ne peut faire l'économie de croiser l'histoire de la représentation en art et qu'elle rejoint, par là, la réflexion sur le sujet moderne.

L'œuvre géographique de Franz Schrader fournit un bon exemple pour aborder ces thèmes de recherche. Quoique occupant peu de place dans les publications d'histoire de la géographie, situé en marge des grandes écoles de pensée qui institutionnaliseront cette discipline au tournant du siècle dernier, Franz Schrader (18441924) reste difficile à placer dans le mouvement des idées. Quand il est mentionné, c'est en général à un ou plusieurs des titres suivants: topographe; cartographe; géographe; auteur de manuels, d'atlas, mais aussi de panoramas; responsable du Bureau cartographique de la maison d'édition Hachette; cousin et proche des frères Reclus; membre éminent de la Société de Géographie de Paris, du Club Alpin Français ou encore de la Société des Peintres de Montagne; partisan du mouvement de protection de la nature; inventeur de l'orographe, etc. Sa contribution apparaît comme trop éclatée pour occuper une place distinctive au sein de l'histoire des catégories établies de la connaissance, quoique le souci de faire des liens entre certains de ses aspects se soit manifesté récemment ( $c f$. BROC, 1974; BERDOULAY, 1995; BERGER-VERDENAL, 1995; SAULE-SORBE, 1997). Il s'agissait pourtant d'une forte personnalité dont l'œuvre, multiple effectivement dans ses manifestations, ne pouvait que trouver sa cohérence dans la démarche qui l'animait. A l'heure où existe une volonté de sortir l'histoire de la géographie des carcans dans lesquels elle s'est trop longtemps trouvée, il est intéressant de se pencher sur le cas de Schrader, tant il transcende les cloisonnements disciplinaires trop souvent pris pour acquis. Nous retiendrons surtout ici que, géographe et cartographe, Schrader est aussi un peintre de talent qui métisse sans arrêt les apports de la science et de l'art. Les pages qui suivent s'attacheront donc à tirer parti des entrecroisements entre ces domaines cultivés le plus souvent séparément.

Tout particulièrement, l'activité incessante de Schrader comme homme de terrain, à la fois topographe, peintre et excursionniste, permet d'étudier la mobilité du regard sous l'angle du déplacement physique de l'observateur, mais aussi du déplacement des concepts, idées et méthodes d'un domaine de la connaissance à un autre. C'est selon cette double perspective que l'on s'interrogera ici. Euvrant dans des domaines traités 
séparément en histoire sociale ou en histoire des sciences et des techniques, Schrader offre l'occasion de s'interroger sur la façon dont le géographe a pu les traverser, voire les réunir.

\section{LE PRIMAT DU REGARD AU XIX SIÈCLE}

La question du regard traverse toute la pensée du XIX ${ }^{\mathrm{e}}$ siècle; il permet ainsi à la réflexion de passer du domaine de l'art à celui de la science, et inversement. Bien évidemment, en géographie, c'est le paysage - vu et représenté - qui est le plus souvent convoqué à ce propos. Nous nous bornerons ici à évoquer trois grands thèmes, qui se recoupent, mais qui aident à cerner la question du regard en rapport avec les préoccupations du XIX ${ }^{\mathrm{e}}$ siècle. Les rappeler brièvement permettra ensuite de mieux apprécier leur portée géographique, et ce, au moyen de l'examen de la contribution de Schrader.

Tout d'abord, il faut mentionner que l'importance accordée à la mobilité du regard ne fait que s'accroître au cours du $\mathrm{XIX}^{\mathrm{e}}$ siècle. Cet intérêt correspond à une des conséquences de la remise en question, commencée bien avant, de la perspective et portant d'abord sur la fixité du regard que celle-ci imposait. La vogue du voyage et sa généralisation à des couches de plus en plus grandes de la population, comme en Europe à partir de la fin du XVIII ${ }^{\mathrm{e}}$ siècle, encouragent une diversification des points de vue sur le monde et des façons - scientifiques, littéraires ou artistiques - dont on cherche à en rendre compte. Tout particulièrement, l'excursion en montagne, par l'immersion qu'elle provoque du sujet dans la nature, contribue à mettre fin à l'immuabilité du point de vue tel que consacré par la perspective.

Apparaissent alors, dès le début du $\mathrm{XIX}^{\mathrm{e}}$ siècle, des tentatives de bousculer les règles qui régissent le paysage "composé". Agrémenté de ruines gréco-romaines et pittoresques, celui-ci obéissait totalement aux lois de la perspective atmosphérique, chromatique et linéaire, faisant d'un seul cas de figure une généralité applicable en tous temps et en tous lieux. Les tentatives nouvelles privilégient très tôt une approche fragmentaire, métonymique, du paysage: on n'en représente plus qu'un morceau, arraché à ce qui l'entoure. Le cadrage, qui lui correspond, implique une part très active du sujet artiste en amont de la production de l'œuvre: c'est par le biais du déplacement du corps et du déplacement du regard que s'opère la sélection (portion, point de vue, distance, angle de vue) qui donnera lieu à son enregistrement. Ces tentatives prendront cependant du temps à s'imposer, tant le regard du récepteur est habitué à la composition classique du paysage. Il faudra attendre le milieu du siècle pour que, malgré les réticences, s'impose la nouvelle approche du paysage, telle que diffusée par l'école de Barbizon, Théodore Rousseau ou Daubigny, ou encore Courbet. Celui-ci n'est-il pas connu pour ses boutades, du type: "où je me place est indifférent"; "tout endroit est bon du moment que j'ai la nature sous les yeux"?

On touche ici au deuxième grand thème qu'il nous faut rappeler, à savoir que l'importance accordée à la vue comme instrument de connaissance au XIX ${ }^{\mathrm{e}}$ siècle traverse d'un même mouvement, les réunissant, la pensée scientifique et la réflexion sur les pratiques artistiques. Goethe, Paester, Alexandre de Humboldt l'illustrent éloquem- 
ment (RECHT, 1989). Mais on aurait tort de confiner cette posture à un courant particulier, le Romantisme ou la Naturphilosophie. Elle va innerver tout le XIX $\mathrm{X}^{\mathrm{e}}$ siècle, au point qu'il serait erroné, par exemple, d'opposer la science - si glorifiée par le positivisme triomphant au cours du siècle - à la recherche artistique, même si l'autonomie de celle-ci sera de plus en plus défendue: on passera d'une association intime de l'art et de la science (comme dans la Naturphilosophie) à une complémentarité plus lâche. Tout particulièrement, l'ombre de Humboldt continuera à peser sur la démarche des géographes jusqu'au début du $\mathrm{XX}^{\mathrm{e}}$ siècle.

Corrélativement, dès la fin des années 1830, des peintres tels que Théodore Rousseau et, à sa suite, Daubigny exécutent de plus en plus l'intégralité de leur toile en extérieur. Ce souci de vérité - le tableau ne peut plus être composé de schèmes préalablement établis - qui les conduit à sortir de l'atelier, se poursuivra tout au long du siècle. La mobilité du regard liée au déplacement du peintre cherche aussi à faire écho à la non permanence de ce qu'il voit. Seule la multiplication des études in situ permettra de saisir la réalité dans ses aspects changeants, voire éphémères, comme l'illustrent bien les célèbres séries Les Meules ou La Cathédrale de Rouen de Claude Monet. Pour un artiste comme Edouard Manet, il s'agit de voir, et non plus de savoir ("savoir" renvoyant ici à un ensemble de données et de principes, hérité et souvent périmé). Marcel Proust, amateur perspicace et attentif de la peinture impressionniste, à travers l'explicitation de la démarche d'Elstir, le peintre d'A la Recherche du Temps Perdu, nous dit bien à quel point ce que nous savons altère ce que nous voyons (Proust, 1918). Autrement dit, le savoir n'est plus en amont du regard, il est en aval et il n'est plus de l'ordre de la projection, mais de l'ordre de la perception. On n'est pas loin du souci de la phénoménologie de dépasser les catégories traditionnelles de la connaissance, c'est-à-dire d'abolir tous les filtres sociaux, culturels ou intellectuels nous séparant de la réalité.

Le troisième thème découle du précédent: c'est celui de la prise de conscience que la mobilité du regard dépend des langages, des outils ou des techniques qui sont utilisés dans l'effort de compréhension du monde. Quand les Impressionnistes mettent en avant la relativité de la vision, de la perception et de la sensation tout à la fois, on constate que se profile, en aval de la relativité, la subjectivité du peintre. En effet, et paradoxalement, l'observation forcenée du paysage amène la peinture à proposer un écart de plus en plus important entre le référent et sa transcription. Dans la complexité des phénomènes perçus et traduits, la solidité de l'objet se dilue dans la singularité d'une approche qui tente de saisir l'insaisissable. L'objet devient un ensemble d'aspects mouvants dont la variété égale celle des observateurs possibles. En étant à l'écoute de la plus absolue de ses sensations visuelles, le peintre prend conscience de la nature éminemment créatrice de l'acte de peindre.

En s'éloignant progressivement d'une représentation liée à un effet de trompel'œil, la peinture de paysage ne craint plus d'afficher les moyens techniques de sa propre production. Un peu comme le géographe explicite les méthodes liées à sa description du monde. On le comprend, l'irruption de la photographie a encouragé ce 
mouvement. Bien accueillie comme atout supplémentaire pour le regard (tel Humboldt qui vantera les perspectives ouvertes par le procédé de Daguerre), la photographie va non seulement encourager le regard parcellaire et métonymique imposé par le cadrage, mais aussi et surtout la prise de conscience de la dépendance du regard à l'égard de la dimension technique et matérielle du langage artistique employé.

C'est en rapport avec ce contexte intellectuel et artistique, à propos des thèmes qui viennent d'être évoqués, que l'exemple de Franz Schrader apporte des éclairages tout-à-fait intéressants sur l'enjeu de la mobilité du regard.

\section{LE DÉPLACEMENT COMME MÉTHODE}

$\mathrm{Au}$ titre de la formulation géographique des expériences liées au déplacement, le cas de Franz Schrader est en effet exemplaire. Celui qui fut connu en partie pour ses travaux de géographie s'oriente vers cette discipline sous l'impulsion de la découverte des Pyrénées. Un matin d'août 1866, depuis Pau où il est arrivé la nuit précédente, Schrader aperçoit les montagnes; le front étiré et azuré de la chaîne des Pyrénées le laisse muet et "stupide devant cette chose nouvelle qui vient d'entrer dans sa vie et qui va la transformer" (HEID, 1936, p. XVI). Dès lors, il n'a de cesse d'y retourner, de mettre des savoirs acquis en autodidacte au service de son étude approfondie. Le massif calcaire de Gavarnie et du Mont-Perdu, dont la cartographie est imprécise, devient rapidement "sa chose". La vulgarisation de ses premiers travaux en 1874 (Carte du Mont-Perdu et de la région calcaire des Pyrénées centrales) coïncide avec les lendemains de la défaite face à l'Allemagne; l'armée française doit se doter de cartes détaillées et fiables sur les zones frontières. Schrader, dont le capitaine du génie Prudent (du Service Géographique de l'Armée) a rapidement repéré les talents et les compétences, va faire partie d'un petit noyau de topographes volontaires et va œuvrer à la cartographie des Pyrénées espagnoles. C'est dans ce cadre-là, celui de déplacements répétés en altitude et en des territoires à l'orographie complexe qu'il va concevoir de nouveaux outils de saisie topographique et développer une approche du paysage montagnard qui opère d'incessants passages entre science et art.

Mobilité physique et mobilité interdisciplinaire sont chez lui indissociables. Il y a d'abord un déplacement initial, le déplacement linéaire du citadin qui va vers la montagne, qui s'extrait du corps social et qui par conséquent se confronte à un univers nouveau pour lui, celui d'une nature sauvage et spécifique. On sait que, selon le projet de la modernité, l'homme a cherché à s'affranchir de cette même nature pour ne plus la subir; mais il peut la retrouver à des fins contemplatives, "à partir du moment où il dispose d'un lieu qui lui fournit un point de vue [sur elle]. Ce lieu, c'est la ville" (RECHT, 1989: 57). Ainsi, Schrader voyage par le train de Bordeaux à Bayonne, d'où il se rend à Pau par voiture. C'est là que la vue sur les Pyrénées introduit une rupture de ses habitudes visuelles. Il suit alors une démarche d'appropriation par la mobilité, réitérant les déplacements en altitude selon le mode rayonnant qui consiste à exploiter toutes les possibilités à partir d'un point d'attache, en l'occurrence Gavarnie, mais encore en organisant des déplacements en boucle pour faire le tour d'un massif. 
En fait, Schrader procède par aires concentriques juxtaposées ou imbriquées. Depuis chaque sommet, alors que par sa position physique le corps domine l'objet d'étude, le géographe repère autour de lui les belvédères qui lui permettront de couvrir à nouveau la zone qu'il étudiera selon d'autres points de vue. Cette stratégie met donc en jeu un autre type de déplacement: celui du regard, qui balaye le tour d'horizon sur $360^{\circ}$, d'un observateur tournant sur lui-même ou se déplaçant dans l'aire restreinte du sommet. C'est la vision "panoramique". C'est sur le cumul de tels déplacements que s'appuie la mobilité du regard chez Schrader.

Il est ici important de souligner un des effets de cette mobilité. Activité motrice continue, le déplacement relie un point à un autre, une activité à une autre. Il est une liaison développée dans la durée, montrant les relations spatiales entre les éléments, les sommets, les crêtes, les dépressions. Il permet de saisir la cohérence d'un ensemble, aussi vaste et complexe soit-il. Pris dans un autre de ses sens (déplacé: qui n'est "pas à sa place"), le déplacement autorise aussi le transfert d'outils, de codes, d'un domaine de la connaissance à un autre. Ce double déplacement nourrit la mobilité du regard de Schrader et est à la source de sa production cartographique. Celle-ci métisse constamment approche artistique et approche scientifique, représentation géographique et représentation artistique, pour le bénéfice de l'une et de l'autre. C'est sur ces déplacements, ces connexions qu'il a profondément vécues et mises en œuvre, que Schrader bâtit sa démarche géographique, en tant que graphie de la terre.

A la clé ou plutôt à la source de ces liens indéfectibles, de ce va-et-vient de la ville à la montagne et d'un champ disciplinaire à l'autre, il y a une dynamique relevant du sensible: l'émotion esthétique, violente et forte. Vécue dans l'instant de la découverte exploratoire, elle trouve un prolongement à travers la déclinaison de pratiques visant à se saisir de son objet. Ainsi, l'orographe, outil qu'il invente (et sur lequel nous reviendrons ci-après), lui permet selon ses propres termes "d'emporter dans [son] sac, non pas des chiffres ou des notes, mais la chaîne de montagnes elle-même", ultime déplacement résultant de tous les autres (SCHRADER, 1875:13). L'instant du ravissement et de la contemplation passé, sans perdre de temps, Schrader se met au travail. La vue, comme sens actif pour Schrader, comme pour Humboldt avant lui, œuvre à la croisée de la jouissance esthétique et de l'élaboration du savoir (cf. HUMBOLDT, 1846-51; SCHRADER, 1898). Par cette importance accordée à la vue dans la démarche de connaissance, mais aussi à la convergence du sentiment esthétique et du travail scientifique, Schrader s'inscrit dans la continuité des postures d'acquisition de connaissance au cours du XIX ${ }^{e}$ siècle et ci-devant mentionnées. Ses travaux sont la concrétisation du primat accordé au regard: le "regard sensible peut être considéré comme l'équivalent du regard logique et de la connaissance spirituelle qui est propre à la raison" (selon Paester, cité par RECHT, 1989:18). Pour Schrader comme pour Humboldt, lorsque la nature devient objet de science, la médiation esthétique de la poésie et de l'art figuratif sont bénéfiques. Ils sont mieux à même de saisir ce qui fait la complexité d'un paysage ou d'un milieu. La description, qu'elle soit littéraire ou graphique et artistique, est le produit d'une faculté "imageante" (ibid., p. 22): c'est ce 
que maitrise Franz Schrader, en cultivant la mobilité de son regard sur le monde et sur les disciplines qui permettent de l'instituer.

\section{LA TECHNIQUE DU TOUR D'HORIZON}

En valorisant la mobilité du regard dans sa démarche, Schrader s'attache à résoudre les nombreux problèmes pratiques liés à son instrumentalisation. Il mobilise toutes les techniques ou outils disponibles pour enregistrer ce qu'il voit lors de ses déplacements: dessins et aquarelles, huiles, notations et croquis dans de petits calepins, chambre noire (jusqu'aux années 1880), appareil photographique à plaques, etc. Il sait aussi, notamment pour sa carte du Mont-Perdu de 1874 et ses premières publications, se faire l'interprète graveur ou lithographe de ses propres travaux graphiques sur matrice de bois ou de pierre. Cette complémentarité de compétences est relativement exceptionnelle chez une seule personne et nourrit son inventivité: Schrader n'hésite pas à en tirer parti pour déplacer les usages des outils et méthodes pertinents à sa recherche.

La façon dont il investit les petits calepins qui assurent le suivi de ses observations topographiques montre à quel point Schrader passe aisément d'un langage à un autre pour instrumentaliser son regard. Ainsi, dans ses carnets de course, il alterne du dessin de visu traditionnel à l'interprétation directe du terrain en codes cartographiques. Il en est de même pour le passage de l'écrit au dessin, le second éclairant le premier: sur la double page du carnet, une esquisse de paysage fixe le cadre de l'itinéraire livré par les mots et les chiffres (lieux, horaires, relevés barométriques). La photographie s'intègre à la panoplie des compétences techniques mobilisées par Schrader. Elle est un outil complémentaire de saisie du visible, dont les données peuvent être refondues par la suite avec d'autres. Tant que les procédés photographiques manqueront de netteté, Schrader n'hésitera d'ailleurs pas à combiner photographie et esquisse détaillée au crayon et à l'aquarelle.

Mais toute cette instrumentalisation du regard ne se situe pas au cœur de sa préoccupation pour la mobilité. Certes, en changeant sa position, l'observateur choisit le point de vue et le cadrage en fonction desquels il met en œuvre ses instruments. Ceux-ci, en quelque sorte, l'accompagnent lors de ses déplacements mais rendent mal compte de ce qu'apporte le mouvement lui-même. C'est notamment le cas de la vue panoramique, étroitement liée à la rotation de l'observateur sur lui-même. On sait l'engouement que le panorama a connu au XIX ${ }^{\mathrm{e}}$ siècle: dispositif d'attraction et de spectacle, visant à atteindre le simulacre le plus abouti, il donnait au spectateur le sentiment d'être de plain pied dans le réel, grâce au cumul d'une multiplicité de points de vue déroulant, à $360^{\circ}$ autour de lui, un paysage ou une scène (COMMENT, 1993). Schrader est aussi auteur de panoramas: panoramas dépliants publiés dans l'Annuaire $d u$ Club alpin français $(1876,1891)$, mais aussi le Panorama du massif du Mont-Blanc $(15 \mathrm{~m} \times 60 \mathrm{~m})$ peint pour le pavillon et la rotonde du Club alpin français à l'Exposition universelle de 1900 à Paris.

A cet intérêt pour la vue panoramique correspond le défi de mettre au point les instruments qui permettent de l'établir avec exactitude. Surtout, l'intérêt scientifique 
provenant de la capacité à passer de ce type de vision à une représentation cartographique précise, le défi a une dimension technique de premier plan (RODES, 1997). C'est pourquoi il est utile de se concentrer ici sur le recours de Schrader à l'orographe: cet instrument, qu'il a inventé, symbolise tous les déplacements physiques, méthodologiques, scientifiques et artistiques que la mobilité du regard met en jeu. Rendu en haut d'un sommet, pouvant tourner sur lui-même, Schrader cherche à opérer le passage de la vue à la carte. "Nos dessins" - a écrit Schrader évoquant ses campagnes topographiques en compagnie de Léonce et Albert Lourde-Rocheblave - "faits d'abord au simple point de vue du pittoresque, s'élargirent bientôt et devinrent des panoramas circulaires, sur lesquels, pendant les soirées d'hiver, nous nous efforcions, à l'aide des cartes de l'Etatmajor, de discerner les pics ou les vallées que nous avions retracés sans en connaitre les noms. Tout ne concordait pas [...]. Rien cependant ne nous disait que nous n'avions pas raison, et le désir d'en avoir le cœur net nous donna la pensée de contrôler nos dessins, non plus après coup, mais à l'avance, de façon que l'examen de la nature devînt une dernière vérification" (SCHRADER, 1875: 6).

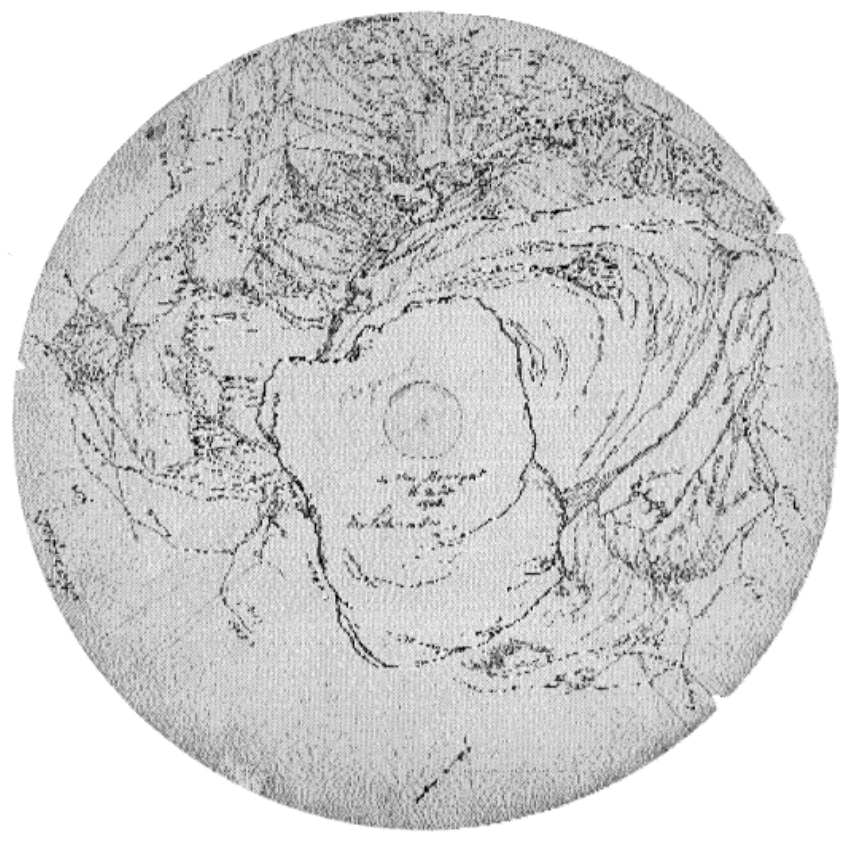

Fig. 1 - Orographie à partir du Pic Mourgat (1906)

Fig. 1 - Orografia vista do Pico Mourgat (1906)

Dans un premier temps, leur méthode demeure rudimentaire. Cela consiste à "prendre un cercle de papier fort, dont le centre est relié à la circonférence par deux minces bandes croisées. Ce centre est fixé sur la carte, à l'aide d'une épingle, en un 
point où nous pensons nous élever. Un fil, attaché à l'épingle et promené sur le contrée environnante, rencontre à des angles divers les cimes ou les vallées dont nous marquons la direction sur le cercle. Ces lignes, transportées à une échelle quelconque sur une large bande de papier, nous donnent autant de verticales, sur lesquelles il est facile de déterminer l'emplacement exact de chaque cime, à l'aide d'un tableau indiquant l'angle d'une différence de hauteur quelconque pour une distance donnée" (ibid., p. 7). Bref, le panorama est appris "théoriquement", par cœur, à Bordeaux, à partir de la carte existante, puis son tracé est confronté à la réalité, sur place, dans les Pyrénées à l'aide du dessin. Erreurs, confusions ou distorsions sont ainsi relevées, mais le point de départ que constitue la carte n'autorise pas l'improvisation au gré des découvertes sur le terrain. Aussi Schrader est-il amené à construire son orographe, instrument adapté au déplacement car il lui permettra "de décalquer l'horizon de chaque point de vue" (ibid., p. 13).

Cette machine à dessiner le relief, apte à relever les terrains complexes, est opératoire en 1873. Elle permet de transcrire directement sur le papier bristol les lignes de paysage qu'il suffit de viser avec sa lunette. Le tracé est direct, instantané. Au fur et à mesure que l'opérateur déplace sa lunette, ses mouvements de visée sont transmis mécaniquement et automatiquement sur une règle équipée d'un crayon. L'opérateur obtient une vue périgraphique anamorphosée sur un disque de $33 \mathrm{~cm}$ : pour chaque sommet où il stationne, Schrader obtient une véritable table d'orientation en modèle réduit (fig. 1). C'est ensuite une construction graphique, en partie contraire aux méthodes mathématiques des géodésiens et topographes, qui permet à Schrader de réaliser sa carte au 1:100 000 en courbes de niveau. La planimétrie est obtenue à partir d'une base mesurée et par triangulation. L'altimétrie découle d'une échelle d'angles verticaux. Les radiales et les calculs se recoupent avec les orographies voisines, dans la logique des déplacements de sommet en sommet déjà évoquée.

Symbole du déplacement nécessaire à l'activité scientifique et artistique, l'orographe correspond à un essai original d'instrumentalisation du regard géographique. Certes, on en comprend facilement la portée pour la cartographie. Mais ne faut-il pas considérer les orographies comme une forme originale d'opérationnalisation d'un des cadres de la description géographique? Camille Vallaux y faisait justement référence en parlant de "tours d'horizon", essentiels pour fournir au géographe les regroupements dont dépend sa démarche descriptive et explicative. Il les définit ainsi: "Les tours d'horizon comprennent tous les ensembles de faits que le géographe peut grouper dans le champ de sa vision, soit réelle, soit idéale, au moins tant que ces faits ne comprennent qu'une partie de la surface terrestre sensiblement circulaire autour de l'observateur et ayant comme limites, soit des lignes de relief aux aspérités variées que l'œil ne peut dépasser, soit la courbure de la surface terrestre matérialisée par d'immenses plaines ou par les eaux de l'Océan" (VALlauX, 1929: 81). Cette idée de tour d'horizon correspond à un souci géographique important dès le XIX $\mathrm{X}^{\mathrm{e}}$ siècle, celui d'une approche régionale de la surface terrestre en tenant compte de la logique du paysage, c'est-à-dire de ce qui est visible. On sait d'ailleurs que le terme de Landschaft 
traduit bien l'ambiguité de ce projet géographique, aux confins de l'analyse régionale et de l'analyse paysagère.

Toutefois, quoiqu'il montre l'importance essentielle du tour d'horizon pour la géographie, Vallaux, pas plus que les autres géographes, ne se penche sur les modalités techniques qui en permettent l'instrumentalisation. En revanche, le soin apporté par Schrader à la mise au point et à l'exploitation de l'orographe démontre, quelles que soient les vertus effectives et les inconvénients de l'appareil, un souci remarquable, et original, de joindre l'intérêt scientifique pour le tour d'horizon aux problèmes liés à son instrumentalisation concrète.

\section{LE PAYSAGE ET LE SUJET}

L'exemple de Schrader montre que la géographie a participé aux grands enjeux qui ont traversé l'art et la science au XIX ${ }^{\mathrm{e}}$ siècle: mobilité du regard, complémentarité des démarches artistiques et scientifiques, prise de conscience des défis techniques pour rendre compte du paysage. C'est que la connaissance géographique n'est pas indépendante des langages qu'elle mobilise (BERDOULAY, 1988). L'intérêt de se pencher sur le cas de Schrader est de montrer que le regard - même et surtout s'il est un cadrage d'une partie de la réalité - ne peut être compris sans les techniques qui le rendent possible et le renouvellent.

Or, l'observateur, dans son rapport au monde qu'il regarde et qu'il décrypte, notamment par le moyen du déplacement dans l'espace terrestre mais aussi dans l'espace interdisciplinaire, prend une part active, en tant que sujet, à l'institution cognitive du paysage. Au déplacement du regard se combinent ainsi celui du sujet et celui des savoirs (y compris techniques) que ce dernier met en jeu. Se déplacer, c'est quitter un point de vue pour en conquérir de nouveaux. Schrader fait même de cette recherche de la nouveauté du regard un principe de méthode. Ainsi, déclare-t-il, pour peindre la montagne, "une première condition, difficile à réaliser, c'est d'oublier ce qu'on a appris en bas, mieux vaudrait même n'avoir rien appris du tout", et, poursuit-il, "quitter le jour de l'atelier ou le plein air des plaines pour arriver devant les blancheurs des hautes cimes, c'est se trouver devant un monde nouveau", (...) où il "faut y devenir un homme nouveau" (SCHRADER, 1998: 21). La modernité, comme recherche de la nouveauté, est au cœur même de sa pratique géographique, à la fois artistique et scientifique.

Autant de positionnements du sujet dans l'espace, autant de points de vue et autant de manières de voir. Ces déplacements se cumulent dans l'expérience du lieu, à travers un redéploiement des descriptions, récits, études, cartes, dessins ou tableaux. Et tout un savoir technique l'accompagne. Même si Schrader n'illustre que le cas particulier du géographe, sa démarche qui valorise le sujet connaissant permet de réfléchir, par extension, aux liens qui se sont tissés, avec la modernité, entre le sujet et le paysage. L'expérience de l'observateur, y compris sa part active, en rapport avec la mobilité du regard induite par les techniques modernes, est un des éléments qui doivent retenir l'attention dans l'étude de l'émergence et de l'évolution du paysage moderne. 
On rejoint alors la réflexion plus générale sur la formation réciproque du sujet et du lieu. En effet, si l'on se concentre sur l'idée de déplacement - celui de l'observateur comme celui des concepts ou méthodes -, la problématique du paysage correspond à celle de la distanciation adéquate à établir de la part du sujet dans son rapport à l'objet et à l'outil. Or c'est bien là que se trouve une des sources vives de la réflexion géographique sur le lieu (BERDOULAY et ENTRIKIN, 1998). En reprenant des termes utilisés par Th. NAGEL (1986), on peut opposer un regard "décentré", que valorise la science positiviste, garante - telle la perspective - de l'objectivité, à un regard "centré" sur soi, refuge de la subjectivité la plus totale. Soucieux de cultiver la mobilité du regard, préoccupé par les apports respectifs de l'art et de la science positive, Schrader a cherché à œuvrer entre cette double polarité, en n'hésitant pas à en tirer parti comme en témoigne la diversité de ses productions. Il nous invite ainsi à souligner l'intérêt de revisiter l'histoire de la pensée géographique à la lumière du thème du regard et de son instrumentalisation, en ouvrant largement la réflexion aux interactions entre l'art et la science, entre le sujet, le paysage et le lieu. L'histoire de la pensée géographique rejoint alors cette géographie culturelle et historique qui cherche à redonner au sujet la place qui lui revient dans la construction réciproque de son identité et des lieux à la surface de la terre.

\section{BIBLIOGRAPHIE}

Berdoulay, V. (1988) - Des mots et des lieux. La dynamique du discours géographique. Ed. du CNRS, Paris.

BERDOULAY, V.(1995) - La formation de l'école française de géographie (1870-1914). $2^{\mathrm{e}}$ ed., Ed. du CTHS, Paris.

Berdoulay, V.; J.N. ENTRIKIN (1998) - Lieu et sujet. Perspectives théoriques. L'Espace géographique, 2, Paris: 111-121.

Berger-Verdenal, M.-G. (1995) - La cartographie des Pyrénées: l'œuvre de Franz Schrader et des topographes du Club alpin français. In V. BERDOUlay (dir.). Les Pyrénées, lieux d'interraction des savoirs (XIX'-début XX $X^{e}$ s.), Ed. du CTHS, Paris: 63-82.

BRoc, N. (1974) - Pour le cinquantenaire de la mort de Franz Schrader. Revue géographique des Pyrénées et du Sud-Ouest, Toulouse: 5-16.

Comment, B. (1993) - Le XIX siècle des panoramas. A. Biro, Paris.

HEID, M. (1936) - Franz Schrader. Esquisse biographique. In Franz Schrader, Pyrénées. Privat, Toulouse, t. 1.

Humboldt, A. de (1846-51) - Cosmos, 3 vol. Gide et Baudry, Paris (ed. original, 1845-47).

NAGEL, T. (1986) - The view from nowhere. Oxford University Press, New York.

Proust, M. (1918) - A l'ombre des jeunes filles en fleurs (A la recherche du temps perdu). N.R.F., Paris.

RECHT, R. (1989) - La lettre de Humboldt. Christian Bourgeois Editeur, Paris.

Rodes, M. (1997) - Le géographe et l'originalité de son apport. In H. SAUle-Sorbe (dir.) infra. 
SAUle-SoRBE, H., dir. (1997) - Franz Schrader, l'homme des paysages rares. 2 t., Ed. du Pin à Crochets, Pau.

SCHRADER, F. (1875) - Etudes géographiques et excursions dans le massif du Mont-Perdu. Gauthier-Villars, Paris.

SCHRAder, F. (1998) - A quoi tient la beauté des montagnes. Ed. du Pin à Crochets, Pau. Réédition de l'article paru dans l'Annuaire du Club alpin français, 1898: 556-577.

VALlAuX, C. (1929) - Les sciences géographiques. $2^{\mathrm{e}}$ ed., F. Alcan, Paris. 